\title{
Protective effects of Lycium barbarum polysaccharide on ovariectomy-induced cognition reduction in aging mice
}

\author{
XIAOMIN ZHENG ${ }^{1,2^{*}}$, JUNYAN WANG $^{2 *}$, FENGCHEN BI $^{2 *}$, YILU LI $^{2}$, JINGJING XIAO $^{2}$, \\ $\mathrm{ZHI} \mathrm{CHAI}^{2}$, YUNHONG LI ${ }^{2}$, ZHENHUA MIAO ${ }^{2}$ and YIN WANG ${ }^{2}$ \\ ${ }^{1}$ Department of Pediatrics, General Hospital of Ningxia Medical University; ${ }^{2}$ Department of Physiology and Neurobiology, \\ Ningxia Medical University, Yinchuan, Ningxia Hui Autonomous Region 750004, P.R. China
}

Received January 5, 2021; Accepted April 8, 2021

DOI: $10.3892 /$ ijmm.2021.4954

\begin{abstract}
Women experience cognitive decline as they age due to the decrease in estrogen levels following menopause. Currently, effective pharmaceutical treatments for age-related cognitive decline are lacking; however, several Traditional Chinese medicines have shown promising effects. Lycium barbarum polysaccharides (LBPs) were found to exert a wide variety of biological activities, including anti-inflammatory, antioxidant and anti-aging effects. However, to the best of our knowledge, the neuroprotective actions of LBP on cognitive impairment induced by decreased levels of estrogen have not yet been determined. To evaluate the effects of LBP on learning and memory impairment in an animal model of menopause, 45 female ICR mice were randomly divided into the following three groups: i) Sham; ii) ovariectomy (OVX); and iii) OVX + LBP treatment. The results of open-field and novel object recognition tests revealed that mice in the OVX group had learning and memory impairments, and lacked the ability to recognize and remember new objects. Notably, these deficits were attenuated following LBP treatment. Immunohistochemical staining confirmed the protective effects of LBP on hippocampal neurons following OVX. To further investigate the underlying mechanism of OVX in mice, mRNA sequencing of the hippocampal tissue was performed, which revealed that the Toll-like receptor 4 (TLR4) inflammatory signaling pathway was significantly upregulated in the OVX group. Moreover, reverse transcription-quantitative PCR and immunohistochemical staining demonstrated that OVX
\end{abstract}

Correspondence to: Professor Zhenhua Miao or Professor Yin Wang, Department of Physiology and Neurobiology, Ningxia Medical University, 1160 Shengli Street, Yinchuan, Ningxia Hui Autonomous Region 750004, P.R. China

E-mail: miaozhenhua2003@163.com

E-mail: yin-wang@hotmail.com

${ }^{*}$ Contributed equally

Key words: Lycium barbarum polysaccharide, cognition reduction, TLR4/NF- $\mathrm{B}$ signaling pathway, neuroinflammation, ovariectomy induced hippocampal injury, upregulated the expression levels of TLR4, myeloid differentiation factor 88 and NF- $\kappa \mathrm{B}$, and increased the expression of TNF- $\alpha$, IL- 6 and IL- $1 \beta$ inflammatory factors. Conversely, LBP treatment downregulated the expression levels of mRNAs and proteins associated with the TLR4/NF- $\mathrm{BB}$ signaling pathway, decreased the inflammatory response and reduced neuronal injury in mice that underwent OVX. In conclusion, the findings of the present study indicated that oral LBP treatment may alleviate OVX-induced cognitive impairments by downregulating the expression levels of mRNAs and proteins associated with the TLR4/NF- $\mathrm{KB}$ signaling pathway, thereby reducing neuroinflammation and damage to the hippocampal neurons. Thus, LBP may represent a potential agent for the prevention of learning and memory impairments in patients with accelerated aging caused by estrogen deficiency.

\section{Introduction}

Aging is a complex process characterized by a progressive loss of functions; in particular, learning and memory impairments (1). In women, decrease in estrogen levels is an important component of the aging following menopause, and brain and endocrine aging occur simultaneously and are closely associated with cognitive impairment and pathological states (2-6).

Inflammation was discovered to be a biomarker of both normal and accelerated aging (7). The cells and tissues of older organisms tend to have higher levels of inflammatory markers, which can lead to low, aseptic, and chronic inflammatory conditions $(8,9)$. This phenomenon has been defined as immune aging and is associated with numerous types of age-related diseases, such as cancer, cardiovascular disease, and neurodegenerative diseases (10-15). Neuroinflammation is a common feature of the majority of central nervous system (CNS) diseases, and has been discovered to cause cognitive impairments $(16,17)$. Aging was reported to influence neuroinflammatory responses by promoting the release of a large number of neuroinflammatory cytokine levels, such as IL-1, IL-6, IL-8, transforming growth factor- $\beta$ (TGF $\beta$ ), TNF- $\alpha$, and activating immune system cells involved in the regulation of neurogenesis, synaptic plasticity, neuronal survival and other critical processes. These changes, in turn, were demonstrated to affect cognitive functions (18-20). 
The effect of estrogen replacement therapy on aging-related changes in cognition remains controversial (2). Therefore, a variety of natural polysaccharides from functional and medicinal foods have attracted considerable attention due to their significant pharmacological activities (21). For example, the polysaccharides of Pleurotus bisporus were reported to have antioxidant and anti-aging effects (22), Sargassum polysaccharide exerted numerous pharmacological activities, including antioxidative proinflammatory, anti-aging and anti-fatigue effects (23) and Cordyceps sinensis polysaccharides were demonstrated to exert significant antioxidant and anti-aging activities (24). As an edible Chinese herbal medicine, Lycium barbarum polysaccharides (LBPs) have been reported to exert antioxidant and neuroimmune regulatory functions $(25,26)$, in addition to anti-inflammatory and anti-aging effects (26-28). However, although the benefits of LBPs have been reported, their effects on learning and memory during the aging process induced by estrogen deficiency are poorly understood, at least to the best of our knowledge.

Ovariectomized (OVX) rodents are useful models for neurocognitive impairments caused by decreased estrogen levels in women (29-31). Hence, the present study aimed to investigate the therapeutic effects of LBP on neuroinflammation and the potential mechanisms underlying its effects using an ovariectomy (OVX) mouse model.

\section{Materials and methods}

Animals studies. A total of 45 female ICR mice (age, 12 weeks; weight, 22-25 g) were purchased from the Laboratory Animal Services Center of Ningxia Medical University [Yinchuan, China; animal license no. SCXK (Ning) 2015-0001]. Mice were housed under standard laboratory conditions at room temperature $\left(22 \pm 2^{\circ} \mathrm{C}\right.$ ) and $30-60 \%$ humidity, with a 12 -h light/dark cycle. Food and water were available ad libitum. Body weight (BW), food and water intake were measured weekly. The present study was conducted in accordance with the 2011 Guidelines for the Protection and Use of Experimental Animals (National Research Council of the National Academies), and approved by the Animal Ethics Committee of General Hospital of Ningxia Medical University, Ningxia, China (approval no. 2020-449).

OVX model induction and LBP treatment. Mice were randomly divided into sham (15 mice) and OVX (30 mice) groups. After adaptive feeding for 1 week, OVX was performed in mice in the OVX group as previously described (32). Briefly, mice were deeply anesthetized with an intraabdominal injection of $50 \mathrm{mg} / \mathrm{kg}$ sodium pentobarbital prior to the operation, and an $\sim 4 \times 4-\mathrm{cm}^{2}$ area of hair was removed from the middle of the back. A 2-3-cm midline incision was made through the skin along the dorsal midline, both ovaries were removed and then the muscle and skin incisions were sutured. Sham mice received anesthesia and underwent the skin and abdominal incision without OVX. Mice were allowed to recover for 7 days prior to further experimentation. OVX mice were subsequently randomly divided into the OVX (15 mice) and OVX + LBP (15 mice; 100 mg/kg/d) (cat. no. RL190914; Xi'an Realin Biotechnology Co., Ltd.; http://ch.xarealin.com/) groups. The mice in the OVX + LBP group received daily intragastric feeding with $10 \mathrm{ml} / \mathrm{kg} \mathrm{LBP}$ solution $(10 \mathrm{mg} / \mathrm{ml})$. Both the sham and OVX groups were administered with $10 \mathrm{ml} / \mathrm{kg} /$ day distilled water. The administration period of this experiment lasted for 24 weeks.

Open field test. All mice were individually placed in the open field (length $\mathrm{x}$ width $\mathrm{x}$ height: $50 \times 50 \times 50 \mathrm{~cm}$ ) for $10 \mathrm{~min}$. The total time taken to enter the central area, the total distance of open land covered and the total number of times the mouse crossed the central square were recorded using an automatic tracking system (Smart 3.0; Panlab; https://www.rwdls.com/). Throughout the experiment, the environmental noise level was $<50 \mathrm{~dB}$.

Novel object recognition test. The experiment was divided into three stages, and each mouse performed the experiment alone. In the first stage, mice were able to move freely in the new object recognition test box for $10 \mathrm{~min}$ and no objects were placed in the test box. In the second stage, the mice were placed into the test box with two identical square objects (A and B), and in the third stage, a square object (B) was replaced with a conical object $(C)$ to recorded as EA (A) and $\mathrm{EB}(\mathrm{B})$ as the total exploration time of the two objects (Fig. 1A). The discriminant index (DI) was calculated using the following formula: $\mathrm{DI}=\mathrm{EB}-\mathrm{EA} /(\mathrm{EA}+\mathrm{EB})$, in which the DI value was $>0.5$ if the mice showed tendencies of exploring novel objects. The preference index (PI), which reflects the ability of mice to explore new objects, was calculated using the following formula: $\mathrm{PI}=\mathrm{EB} /(\mathrm{EA}+\mathrm{EB})$.

Preparation of mouse brain samples. At 36 weeks old, all mice were euthanized with $200 \mathrm{mg} / \mathrm{kg}$ sodium pentobarbital. Then brains were immediately dissected and the hippocampus was isolated. Then, half the brains from each group were fixed in $4 \%$ paraformaldehyde (LEAGENE ${ }^{\circledR}$; http://www.leagene. com/) for $24 \mathrm{~h}$ at $4^{\circ} \mathrm{C}$, dehydrated in an ascending series of alcohol and embedded in paraffin. Paraffin-embedded tissues were cut into $4-\mu \mathrm{m}$-thick sections, washed with $1 \mathrm{X}$ PBS at room temperature and deparaffinized in xylene before undergoing routine histology and immunohistochemical analysis. The remaining brains from each group were preserved in liquid nitrogen for mRNA sequencing, western blotting and reverse transcription-quantitative PCR (RT-qPCR) analysis.

ELISA. Blood was collected from mice in each group into 1.5-ml centrifuge tubes, maintained at room temperature for $2 \mathrm{~h}$ and centrifuged at $1,000 \mathrm{x}$ f for $20 \mathrm{~min}$ at $4^{\circ} \mathrm{C}$ to obtain the serum for ELISA analysis. The concentration of estrogen in the serum of mice was measured using an ELISA detection kit (cat. no. SU-B20462; Quanzhou Konodi Biotechnology Co., Ltd.; http://www.qzkndbio.com/), according to the manufacturer's protocol. The optical density was measured at a wavelength of $450 \mathrm{~nm}$ using a microplate reader (Varioskan ${ }^{\mathrm{TM}}$ LUX; Thermo Scientific ${ }^{\mathrm{TM}}$; Thermo Fisher Scientific, Inc.). Linear regression equations were used to analyze the results.

mRNA library construction and RNA sequencing. Total RNA was isolated and purified using TRIzol ${ }^{\circledR}$ reagent (Invitrogen; Thermo Fisher Scientific, Inc.) according to the manufacturer's protocol. The concentration and purity of RNA from each 
A

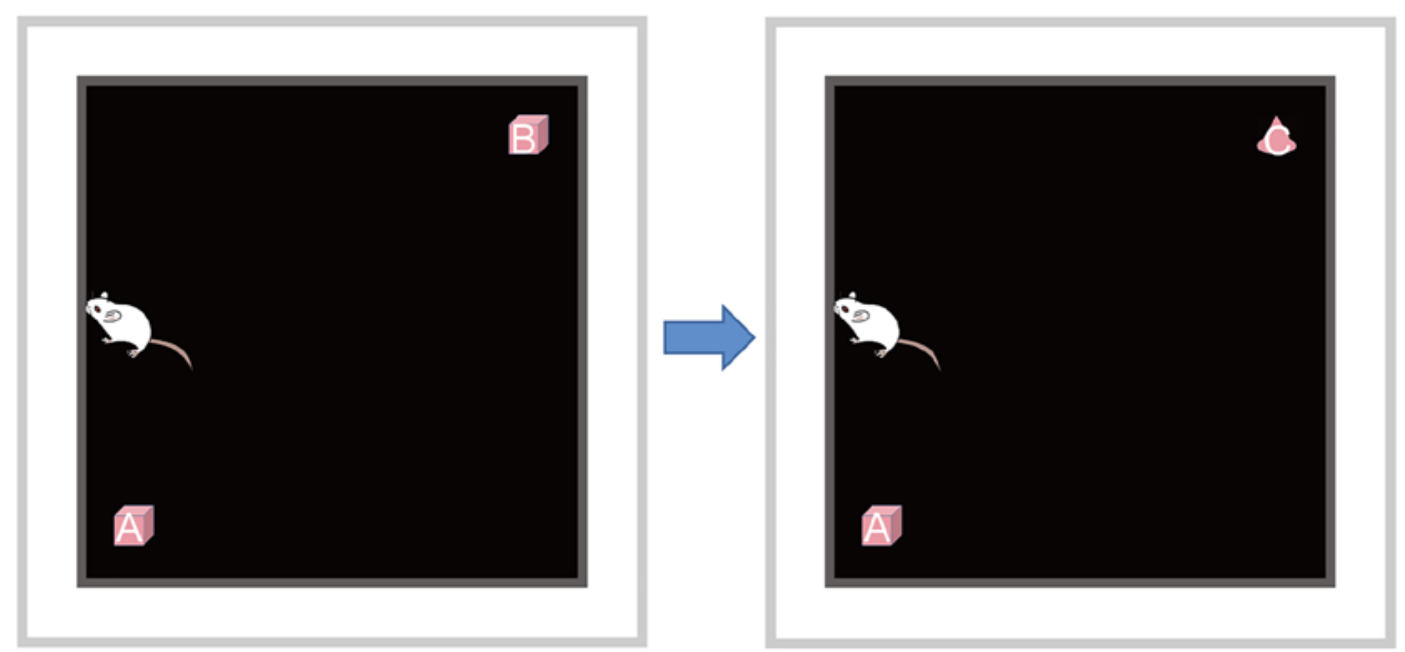

B
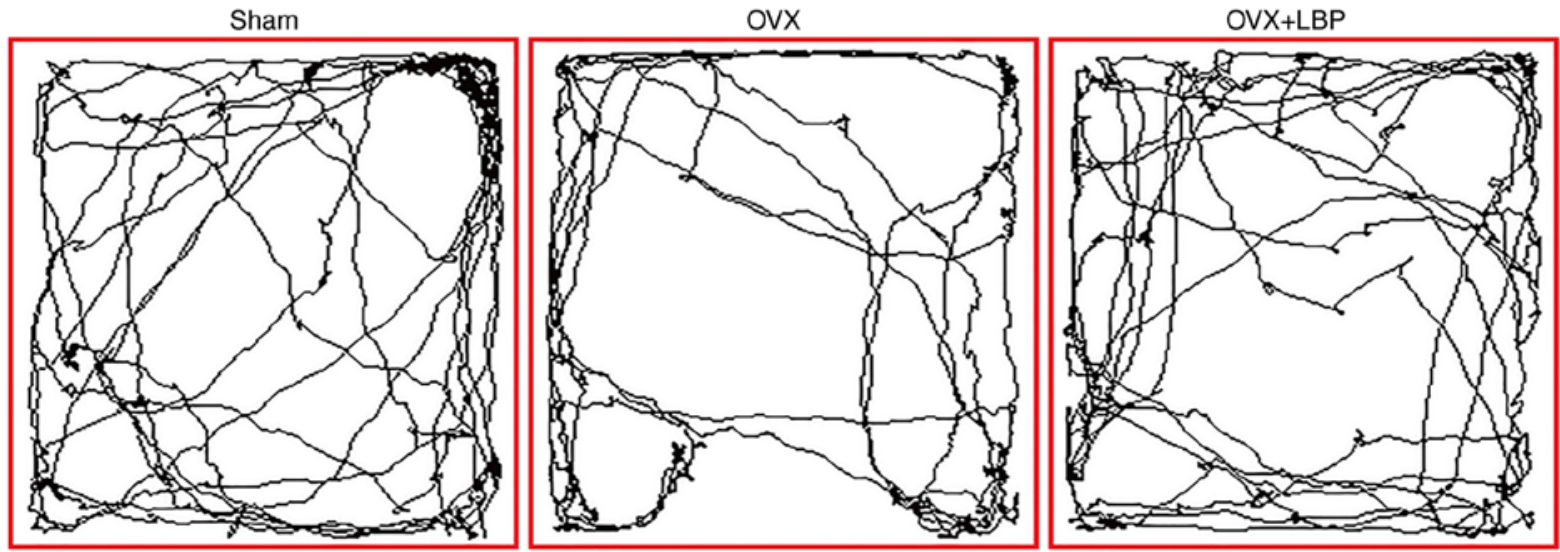

C

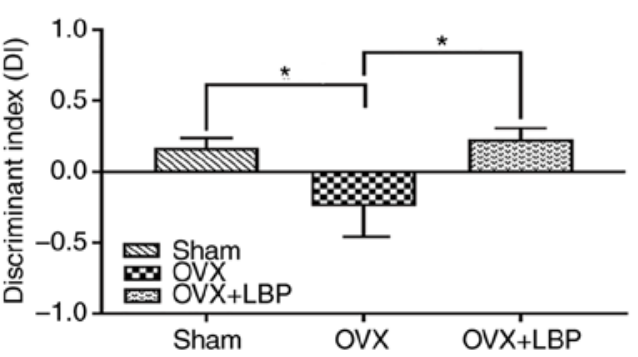

D

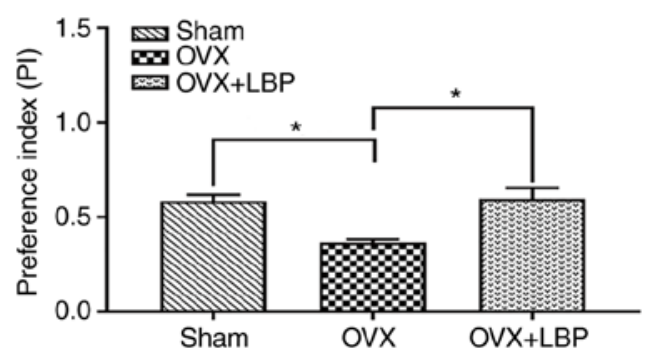

Figure 1. Effects of LBP on non-spatial learning, recognition and memory function in OVX model mice. (A) Schematic diagram of the novel object recognition test. (B) Representative images of motion trajectories in the sham, OVX and OVX + LBP groups. (C) The DI values of the sham, OVX and OVX + LBP groups. (D) The PI values of the sham, OVX and OVX + LBP groups. All data are expressed as the mean \pm SEM of 6-7 mice in each group. Data were analyzed using a one-way ANOVA and an LSD post hoc test. "P<0.05 vs. the OVX group. LBP, Lycium barbarum polysaccharide; OVX, ovariectomy; DI, the discriminant index; PI, the preference index.

sample were quantified using NanoDrop ND-1000 spectrophotometer (Thermo Fisher Scientific, Inc.). RNA integrity was assessed using a Bioanalyzer 2100 (Agilent Technologies, Inc.) with an RNA integrity of $>7.0$, and the findings were confirmed via electrophoresis with denaturing agarose gel. Poly(A) RNA was purified from $1 \mu \mathrm{g}$ of total RNA using Dynabeads Oligo (dT)25-61005 (Thermo Fisher Scientific, Inc.) following two rounds of purification. Then, the poly (A) RNA was fragmented into small pieces using a NEBNext ${ }^{\circledR}$ Magnesium RNA Fragmentation Module (cat. no. e6150; New England BioLabs, Inc.) for 5-7 min at $94^{\circ} \mathrm{C}$. The cleaved RNA fragments were subsequently reverse transcribed into cDNA using SuperScript $^{\mathrm{TM}}$ II Reverse Transcriptase (cat. no. 18064014; Invitrogen; Thermo Fisher Scientific, Inc.) according to the manufacturer's protocol, which was then used to synthesize U-labeled second-stranded DNA with DNA Polymerase I (E. coli) (cat. no. m0209; New England BioLabs, Inc.), RNase H (cat. no. m0297; New England BioLabs, Inc.) and dUTP solution (cat. no. R0133; Thermo Fisher Scientific, Inc.). An A-base was then added to the blunt ends of each strand to prepare them for ligation to the 18064014 indexed adapters; each adapter contained a T-base overhang for ligating the adapter to the A-tailed fragmented DNA. Single- or dual-index adapters were ligated to the fragments, and size selection was performed using AMPureXP beads (cat. no. A63881; Beckman Coulter, Inc.). After the U-labeled second-stranded DNA was treated with a heat-labile UDG enzyme (cat. no. m0280; New England BioLabs, Inc.), the ligated products were amplified 
via PCR using the following thermocycling conditions: Initial denaturation at $95^{\circ} \mathrm{C}$ for $3 \mathrm{~min}$, followed by eight cycles of denaturation at $98^{\circ} \mathrm{C}$ for $15 \mathrm{sec}$, annealing at $60^{\circ} \mathrm{C}$ for $15 \mathrm{sec}$ and extension at $72^{\circ} \mathrm{C}$ for $30 \mathrm{sec}$; and a final extension at $72^{\circ} \mathrm{C}$ for $5 \mathrm{~min}$. The average insert size for the final cDNA library was $300 \pm 50$-bp. Finally, the $2 \times 150$-bp paired-end sequencing (PE150) was performed on an Novaseq ${ }^{\mathrm{TM}} 6000$ system (Illumina, Inc.) according with the manufacturer's protocol.

Cutadapt software (https://cutadapt.readthedocs. io/en/stable/, version:cutadapt-1.9) was used to remove reads that contained adaptor contamination (command line: cutadapt -a ADAPT1 -A ADAPT2 -o out1.fastq-p out2.fastq in1.fastq in2.fastq -O 5-m100). After removing low-quality and undetermined bases, HISAT2 software (https://daehwankimlab.github.io/hisat2/; version, hisat2-2.0.4) was used to map reads to the genome (for example, Homo sapiens Ensembl v96; command line: hisat2 -1 R1.fastq.gz -2 R1.fastq.gz -S sample_mapped.sam). The mapped reads of each sample were assembled using StringTie (http://ccb.jhu.edu/software/stringtie/; version, stringtie-1.3.4d.Linux_x86_64) with default parameters (command line: $\sim$ stringtie-p4-G genome. gtf-o output. gtf-l sample input. bam). Then, transcriptomes from all samples were merged to reconstruct a comprehensive transcriptome using gffcompare software (http://ccb.jhu. edu/software/stringtie/gffcompare.shtml; version, gffcompare-0.9.8. Linux_x86_64). After the final transcriptome was generated, StringTie and ballgown (http://www.bioconductor.org/packages/release/bioc/html/ballgown.html) were used to estimate the expression levels of all transcripts and to determine mRNA expression based on fragments per kilobase of exon (FPKM) using the following equation: FPKM = [total_exon_fragments/mapped_reads (millions) $\mathrm{x}$ exon_length $(\mathrm{kB})$; command line: $\sim$ stringtie- -B-p4-G merged.gtf-osamples.gtf samples.bam]. Differentially expressed genes (DEGs) with fold changes of $>2$ or $<0.5$ and $\mathrm{P}<0.05$ were selected using the $\mathrm{R}$ package, edge $\mathrm{R}$ (https://bioconductor.org/packages/release/bioc/html/edgeR. html) or DESeq2 (http://www.bioconductor.org/packages/release/bioc/html/DESeq2.html) $(33,34)$. Gene Ontology (GO) (35) functional term and Kyoto Encyclopedia of Genes and Genomes (KEGG) (36) signaling pathway enrichment analyses were then used to evaluate DEGs.

$R T$-qPCR. Total RNA from hippocampal tissues was extracted using TRIzol ${ }^{\circledR}$ reagent (Invitrogen ${ }^{\mathrm{TM}}$; Thermo Fisher Scientific, Inc.). Total RNA was reverse transcribed into cDNA using a PrimeScript ${ }^{\mathrm{TM}}$ RT reagent kit (cat. no. RR047A; Takara Biotechnology Co., Ltd.) at $37^{\circ} \mathrm{C}$ for $15 \mathrm{~min}$ and $85^{\circ} \mathrm{C}$ for $5 \mathrm{sec}$, and then maintained at $4^{\circ} \mathrm{C}$. qPCR was performed using a PerfectStart ${ }^{\mathrm{TM}}$ Green qPCR Super Mix (cat. no. AQ601; Beijing TransGen Biotech Co., Ltd.) on a CFX96 ${ }^{\mathrm{TM}}$ Real-Time PCR detection system (Bio-Rad Laboratories, Inc.). The following thermocycling conditions were used for the qPCR: Initial denaturation at $94^{\circ} \mathrm{C}$ for $30 \mathrm{sec}$, followed by 40 cycles of annealing at $94^{\circ} \mathrm{C}$ for $5 \mathrm{sec}$, and extension at $60^{\circ} \mathrm{C}$ for $30 \mathrm{sec}$. GAPDH was used as the endogenous reference gene for normalizing Toll-like receptor 4 (TLR4), myeloid differentiation factor 88 (MyD88), NF- $\mathrm{B}$, IL-1 $\beta$, IL-6 and TNF- $\alpha$ expression in the mouse hippocampus. The sequences of the primers used for the qPCR are presented in Table I. The expression levels were quantified using the $2^{-\Delta \Delta \mathrm{Cq}}$ method (37).

Histopathological analysis. The paraffin sections were cut to a thickness of $4-\mu \mathrm{m}$, stored at $60^{\circ} \mathrm{C}$ for $2 \mathrm{~h}$ and then deparaffinized with xylene at room temperature. The sections were subsequently rehydrated with a descending alcohol series at room temperature (anhydrous ethanol I, $5 \mathrm{~min}$; anhydrous ethanol II, $5 \mathrm{~min}$; 95\% alcohol, $5 \mathrm{~min}$; $85 \%$ alcohol, $5 \mathrm{~min}$; and $75 \%$ alcohol, 5 min). The sections were hydrated with tap water for $3 \mathrm{~min}$ and then stained with a hematoxylin and eosin (H\&E) staining kit (cat. no. DH0006; LEAGENE ${ }^{\circledR}$; http://www. leagene.com/). Briefly, the sections were stained with hematoxylin for $5 \mathrm{~min}$ at room temperature, washed with tap water for $10 \mathrm{~min}$, differentiated with $1 \%$ hydrochloric acid alcohol differentiation for 2-3 sec, washed with water for $15 \mathrm{~min}$ and stained with eosin dye solution for $5 \mathrm{~min}$ at room temperature. The sections were then dehydrated with an ascending gradient alcohol series at room temperature (70\% alcohol, $1 \mathrm{~min} ; 80 \%$ alcohol, $1 \mathrm{~min}$; 90\% alcohol, $1 \mathrm{~min}$; $95 \%$ alcohol, $5 \mathrm{~min}$; anhydrous ethanol I, $10 \mathrm{~min}$; anhydrous ethanol II, $10 \mathrm{~min}$; and ethanol III, $5 \mathrm{~min}$ ) and deparaffinized with xylene (CAS no. 1330-20-7; Tianjin Damao Chemical Reagent Factory) at room temperature (xylene I, $10 \mathrm{~min}$; xylene II, $10 \mathrm{~min}$; and xylene III, $10 \mathrm{~min}$ ). The slices were sealed with neutral balsam (CAS no. 96949-21-2; Beijing Solarbio Science \& Technology Co., Ltd.) and dried in a ventilation cabinet. A 200-fold image of CA1 and CA3 was captured (DP73; Olympus Corporation) to view the organization and structure. Neuronal damage was rated in accordance with this scoring criteria proposed by Shi et al (38).

Nissl staining. Nissl staining was performed to evaluate the extent of neuronal damage in the hippocampus. The paraffin sections of the brain were cut into $4-\mu \mathrm{m}$ thick sections and the methods of deparaffinization, rehydration and hydration were performed as described for H\&E staining. Then, after rinsing the sections with $1 \mathrm{X}$ PBS, the sections were stained with $0.1 \%$ cresol purple dye (CAS no. 10510-54-0; Beijing Solarbio Science \& Technology Co., Ltd.) for $30 \mathrm{~min}$ at room temperature, washed with distilled water and differentiated with $0.5 \%$ acetic acid solution for 2-3 sec (CAS no. 64-19-7; Xiya Reagent $^{\circledR}$ : http://www.xiyashiji.com/) at room temperature. Subsequent steps, including dehydration, xylene transparency and neutral gum sealing were performed as described for $\mathrm{H} \& \mathrm{E}$ staining. Images were captured of CA1 and CA3 (200 and 400-fold) (DP73; Olympus Corporation) to view the organization and structure. The Nissl body counts in the hippocampal CA1 and CA3 regions from each animal were calculated using Image Oro-Plus 6.0 software (Media Cybernetics, Inc.).

Immunohistochemical analysis. Immunohistochemical analyses were performed to analyze the expression of TLR4, MyD88, NF- $\kappa$ B, TNF- $\alpha$, IL- 6 and IL- $1 \beta$ in paraffin-embedded brain tissue sections ( $4-\mu \mathrm{m}$ thick). The methods of deparaffinization, rehydration and hydration of the paraffin sections were performed as described for H\&E staining, and sections were subsequently washed with $1 \mathrm{X}$ PBS. Antigen retrieval was performed via incubation with citrate buffer $(\mathrm{pH} 6.0$; cat. no. s8220; Beijing Solarbio Science \& Technology Co., 
Table I. Sequences of the primers used for reverse transcription-quantitative PCR (5'-3').

\begin{tabular}{lll}
\hline Primer & \multicolumn{1}{c}{ Forward } & \multicolumn{1}{c}{ Reverse } \\
\hline GAPDH & ACAACTTTGGCATTGTGGAA & GATGCAGGGATGATGTTCTG \\
TLR4 & CCGCTTTCACCTCTGCCTTCAC & ACCACAATAACCTTCCGGCTCTTG \\
MyD88 & AGCAGAACCAGGAGTCCGAGAAG & GGGCAGTAGCAGATAAAGGCATCG \\
NF- $\kappa$ p 65 & GACACGACAGAATCCTCAGCATCC & CCACCAGCAGCAGCAGACATG \\
TNF- $\alpha$ & GCCTCTTCTCATTCCTGCTTGTGG & GTGGTTTGTGAGTGTGAGGGTCTG \\
IL-1 $\beta$ & TCGCAGCAGCACATCAACAAGAG & AGGTCCACGGGAAAGACACAGG \\
IL-6 & CTTCTTGGGACTGATGCTGGTGAC & AGGTCTGTTGGGAGTGGTATCCTC
\end{tabular}

TLR, Toll-like receptor.

Ltd.) in a microwave oven for $10 \mathrm{~min}$, the endogenous peroxidase activity was blocked with $3 \% \mathrm{H}_{2} \mathrm{O}_{2}$ (CAS no. 7722-84-1; Aikeshiji) for $10 \mathrm{~min}$ at room temperature and blocking for non-specific binding was subsequently performed with $10 \%$ goat serum (cat. no. SL038; Beijing Solarbio Science $\&$ Technology Co., Ltd.) for $1 \mathrm{~h}$ at room temperature. The brain tissues were then incubated with the following primary antibodies at $4^{\circ} \mathrm{C}$ overnight: Anti-MyD88 (cat. no. WL02494; 1:200), anti-NF-кB (cat. no. WL01980; 1:200), anti-IL-6 (cat. no. WL02841; 1:200), anti-TNF- $\alpha$ (cat. no. WL01896; 1:200), anti-IL-1 $\beta$ (cat. no. WLH3903; 1:200) (all Wanleibio Co., Ltd.) and anti-TLR4 (cat. no. ab22048; Abcam; 1:100). Following the primary antibody incubation, the sections were incubated with HRP-conjugated secondary antibodies (code nos. 115-035-003, 1:200; and 111-035-003, 1:500; Jackson ImmunoResearch Laboratories, Inc.) for $2 \mathrm{~h}$ at room temperature. The sections were then incubated with a DAB staining kit (cat. no. DAB-1031; MXB Biotechnology) and counterstained with hematoxylin for $60 \mathrm{sec}$ at room temperature. Finally, the sections were rinsed in purified water for $60 \mathrm{sec}$ and observed under a light microscope (400-fold) (DP73; Olympus Corporation). Protein expression was calculated using Image pro-Plus 6.0 software.

Western blotting. Total protein was extracted from mouse hippocampal tissue using a total protein extraction kit (cat. no. KGP2100; Nanjing KeyGen Biotech Co., Ltd.). Total protein was quantified using a BCA kit (cat. no. KGP902; Nanjing KeyGen Biotech Co., Ltd.) and 30-50 $\mu \mathrm{g}$ protein per lane was separated via SDS-PAGE using a 10\% PAGE Gel Fast Preparation kit (cat. no. PG112; Shanghai EpiZyme Biotech Co., Ltd.; http://www.epizyme.cn/). The separated proteins were subsequently transferred onto a polyvinylidene fluoride membrane (cat. no. IPVH00010; Immobilon ${ }^{\circledR}$-P; Merck KGaA) and blocked with $5 \%$ skimmed milk powder at room temperature for $2 \mathrm{~h}$. The membranes were incubated with the following primary antibodies overnight at $4^{\circ} \mathrm{C}$ on a shaker: Anti-TLR4 (cat. no. WL00196; Wanleibio Co., Ltd.;

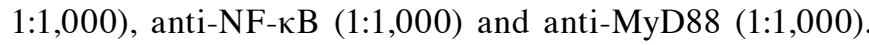
Following the primary antibody incubation, the membranes were incubated with an HRP-conjugated goat anti-rabbit IgG secondary antibody (1:5,000; cat. no. A21030; Abbkine Scientific Co., Ltd.) for $1 \mathrm{~h}$ at room temperature. Protein bands were visualized using an enhanced chemiluminescence reagent (cat. no. 34094; Pierce; Thermo Fisher Scientific, Inc.) and a 600 ultra-sensitive multifunctional imager (Amersham; Cytiva). ImageJ v1.8.0 software was used for densitometric analysis (National Institutes of Health).

Statistical analysis. Each experiment was repeated at least 3 times or using three mice. Statistical analysis was performed using GraphPad 8.0 software (GraphPad Software, Inc.). Statistical differences between groups were determined using a one-way ANOVA and an LSD post hoc test. Data were presented as the mean $\pm \mathrm{SEM} . \mathrm{P}<0.05$ was considered to indicate a statistically significant difference.

\section{Results}

LBP attenuates OVX-induced learning and memory impairments in mice. The novel object recognition test was used to assess non-spatial learning, recognition, and memory function in OVX mice treated with LBP (Fig. 1). Although all three groups (sham, OVX and OVX + LBP) exhibited DI values of $<0.5$, the OVX group exhibited a negative DI value. Mice in the OVX group also displayed a significantly decreased ability to explore new objects and a decreased preference for new objects (therefor, PI) compared with the other two groups. Collectively, these findings indicated that OVX may decrease the ability of the mouse to recognize and remember new objects and that LBP treatment may repair these deficits (Fig. 1B-D).

An open-field test was used to evaluate the effect of LBP on the motor ability of mice in OVX and OVX + LBP groups. The findings revealed that both the number of crossings and total distance traveled were significantly decreased in the OVX group compared with the sham group, suggesting that mice experienced decreased motor ability and an increased fear of the open environment following OVX. By contrast, the number of central crossings and total distance traveled were significantly increased in the OVX + LBP group compared with the OVX group, indicating that LBP treatment may increase motor ability and decrease fear of the open environment in the OVX model mice (Fig. 2B and C).

LBP treatment inhibits hippocampal neuronal damage in OVX model mice. To determine the effects of LBP on OVX-induced hippocampal neuronal injury, H\&E and Nissl 
A

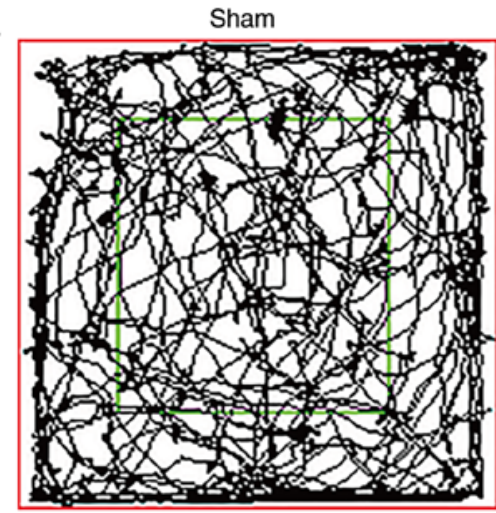

B

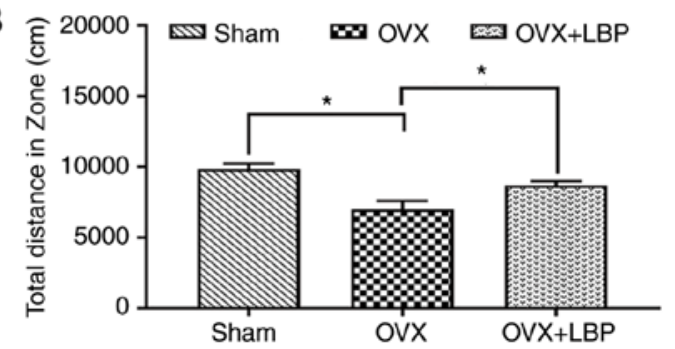

OVX

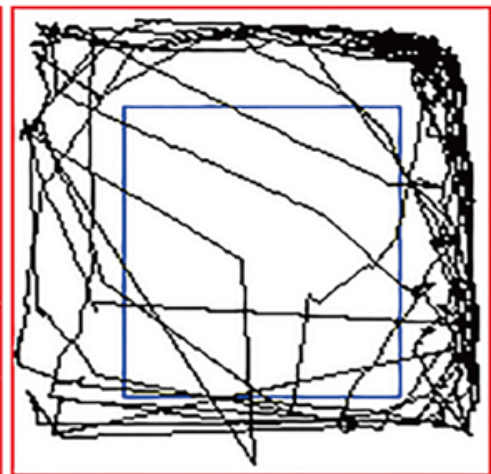

OVX+LBP

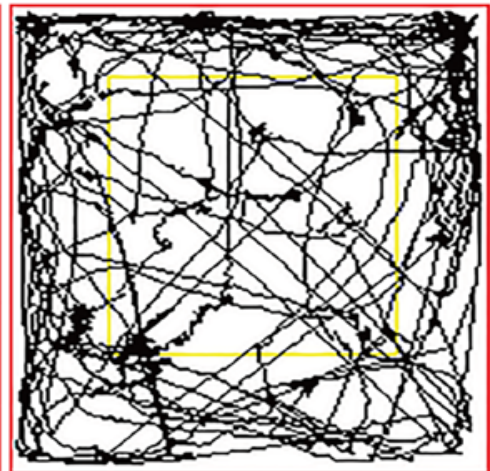

C

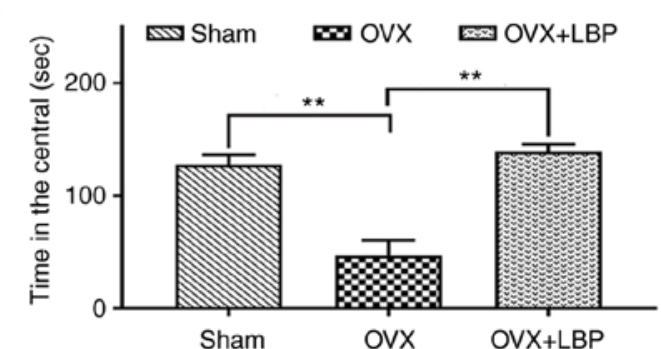

Figure 2. Effects of LBP on the motor ability and anxiety-like behavior of OVX model mice. (A) Representative images of motion trajectories in the sham, OVX and OVX + LBP groups. (B) Total distance traveled during the open field test. (C) Time spent in the center area among different groups. All data are expressed as the mean \pm SEM of 6-7 mice in each group. Data were analyzed using a one-way ANOVA and an LSD post hoc test. ${ }^{*} \mathrm{P}<0.05$ and ${ }^{* *} \mathrm{P}<0.01$ vs. the OVX group. LBP, Lycium barbarum polysaccharide; OVX, ovariectomy.

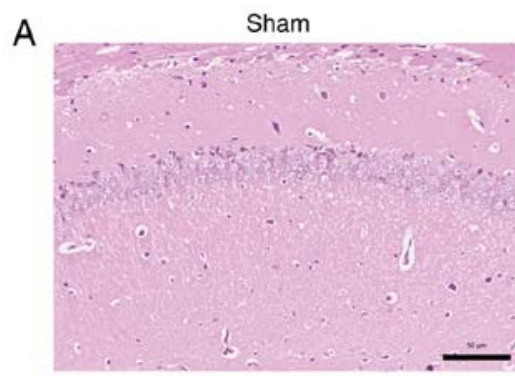

B

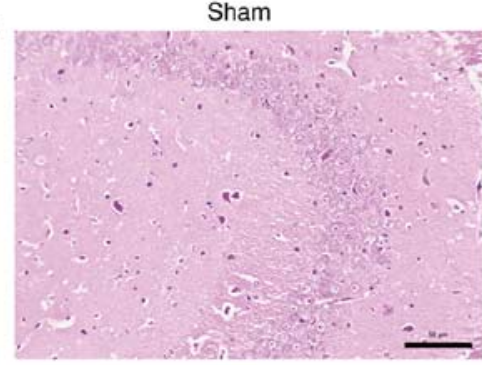

C

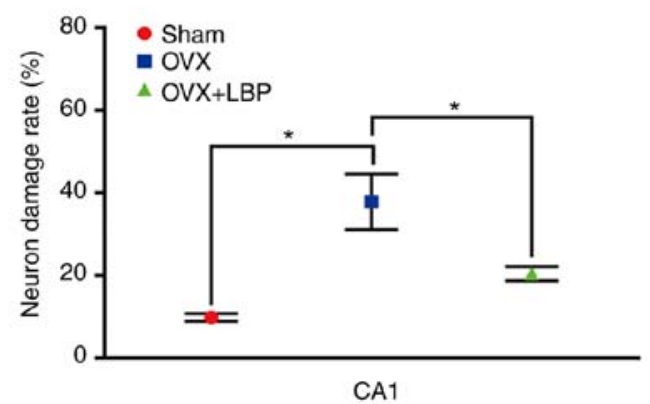

ovx

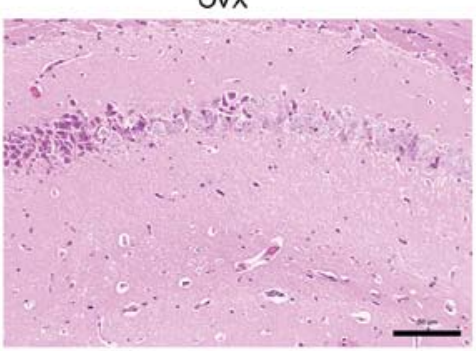

ovx

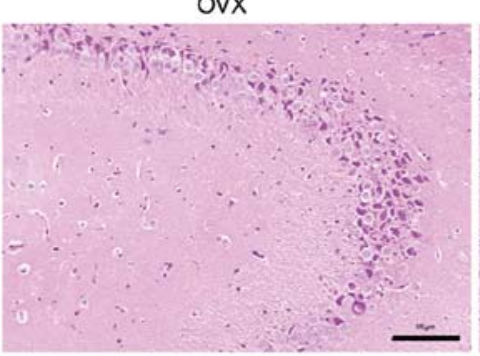

OVX+LBP

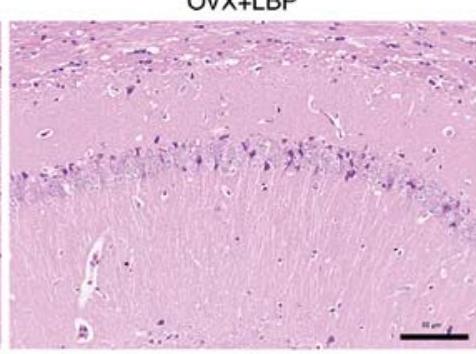

OVX+LBP

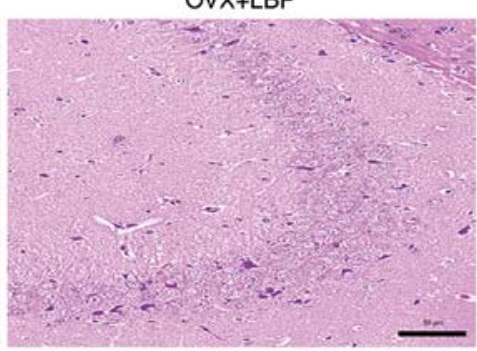

D

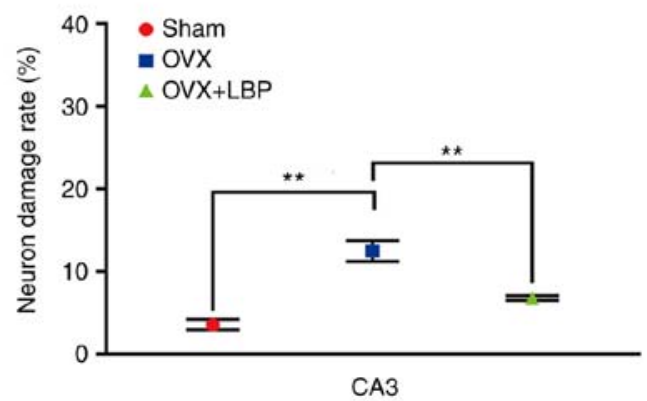

Figure 3. LBP treatment significantly attenuates injury to hippocampal CA1 and CA3 neurons in OVX model mice. (A and B) Hematoxylin and eosin staining was used for the histological examination of neurons in the hippocampal CA1 and CA3 regions. The scale bar was $50 \mu \mathrm{m}$. (C and D) Rates of CA1 and CA3 neuron damage in OVX model mice. Data were analyzed using a one-way ANOVA and an LSD post hoc test. ${ }^{*} \mathrm{P}<0.05$ and ${ }^{* *} \mathrm{P}<0.01 \mathrm{vs.}$. the OVX group (n=5/group). LBP, Lycium barbarum polysaccharide; OVX, ovariectomy. 

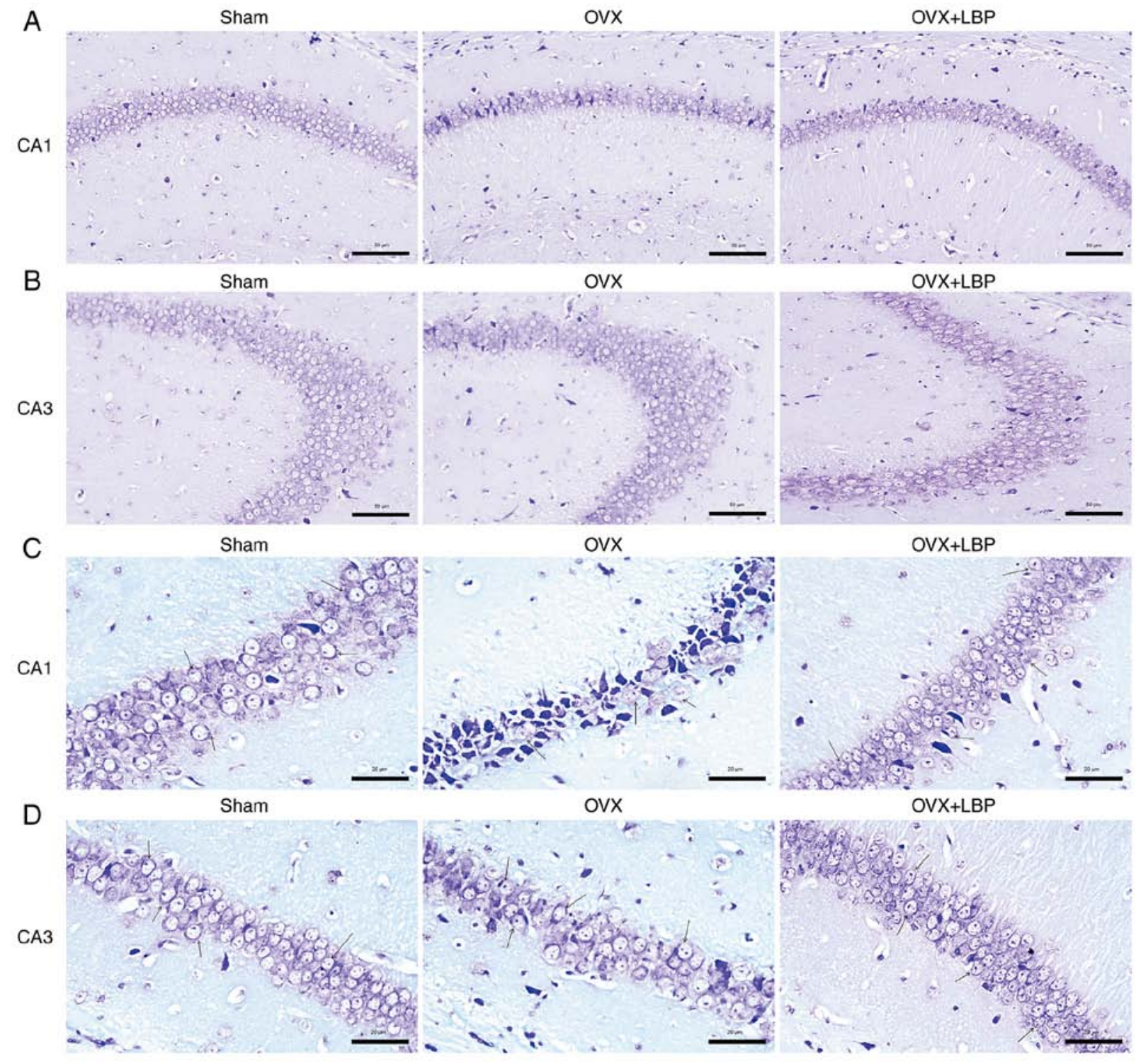

ovx

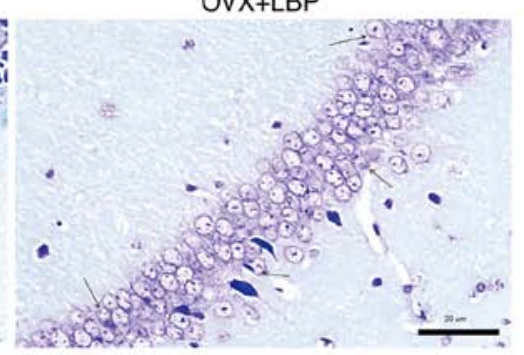

E

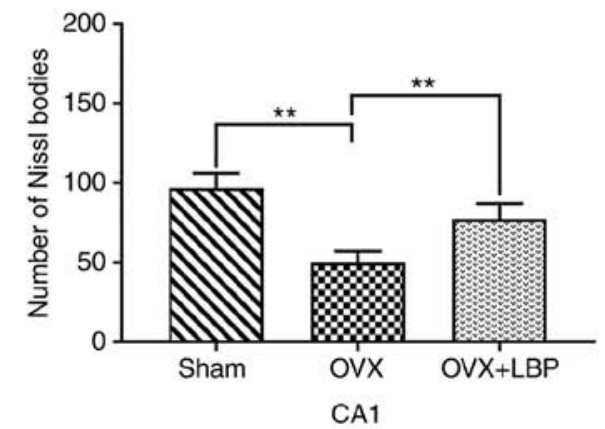

F

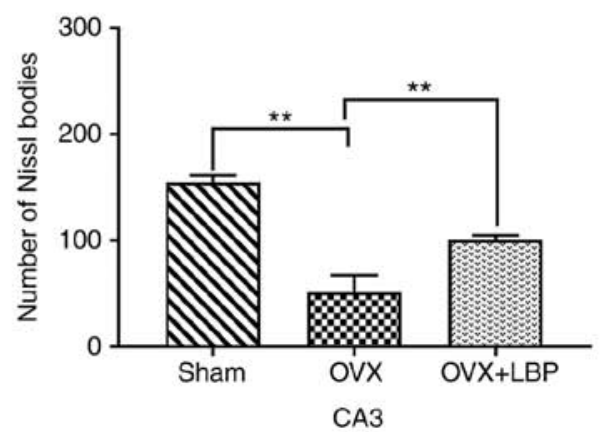

Figure 4. LBP increases the number of hippocampal neurons in OVX mice. (A-D) Nissl staining was used for histological examination of the hippocampal CA1 and CA3 regions. The scale bar was $50 \mu \mathrm{m}$ for A and B and $20 \mu \mathrm{m}$ for C and D. (E and F) Nissl body counts in the CA1 and CA3 regions in OVX mice. Data were analyzed using a one-way ANOVA and an LSD post hoc test. ${ }^{* *} \mathrm{P}<0.01$ vs. the OVX group ( $\mathrm{n}=5 /$ group). LBP. Lycium barbarum polysaccharide; OVX, ovariectomy.

staining were performed. Overt signs of neuronal injury were observed in the hippocampal CA1 and CA3 regions of the OVX model mice; however, these changes were significantly attenuated following LBP treatment (Fig. 3A-D). In addition, Nissl staining revealed significant decreases in the number of Nissl-positive bodies in the hippocampal CA1 and CA 3 regions of the OVX model group compared with the sham group.
However, the number of Nissl bodies in the hippocampal CA1 and CA3 regions was significantly increased following LBP treatment (Fig. 4A-F).

RNA sequencing transcriptional analysis the possible effect of OVX on the hippocampus of mice. To determine the effects of the OVX surgery, RNA sequencing analysis on hippocampal 


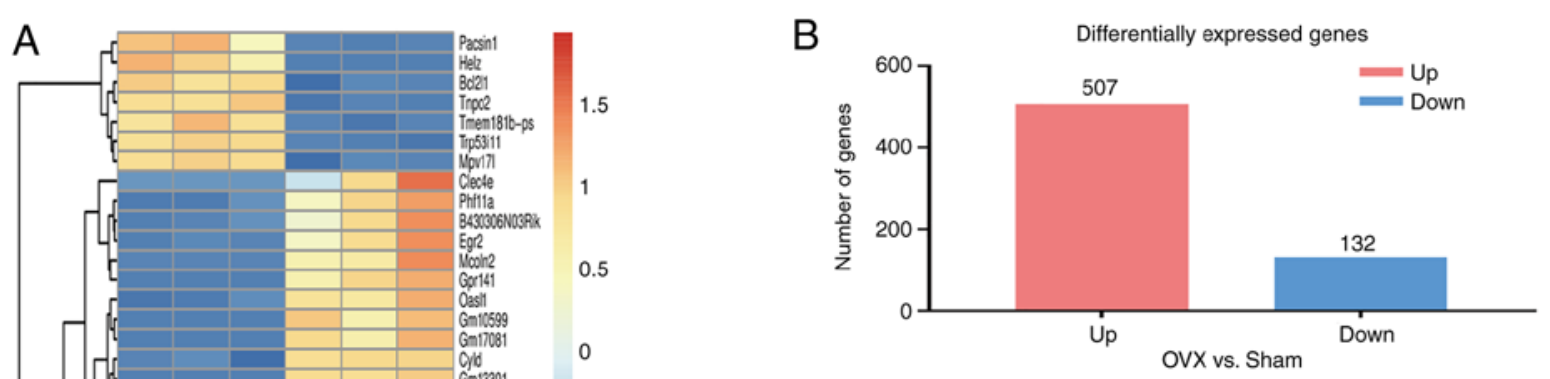

$-0.5 \mathrm{C}$
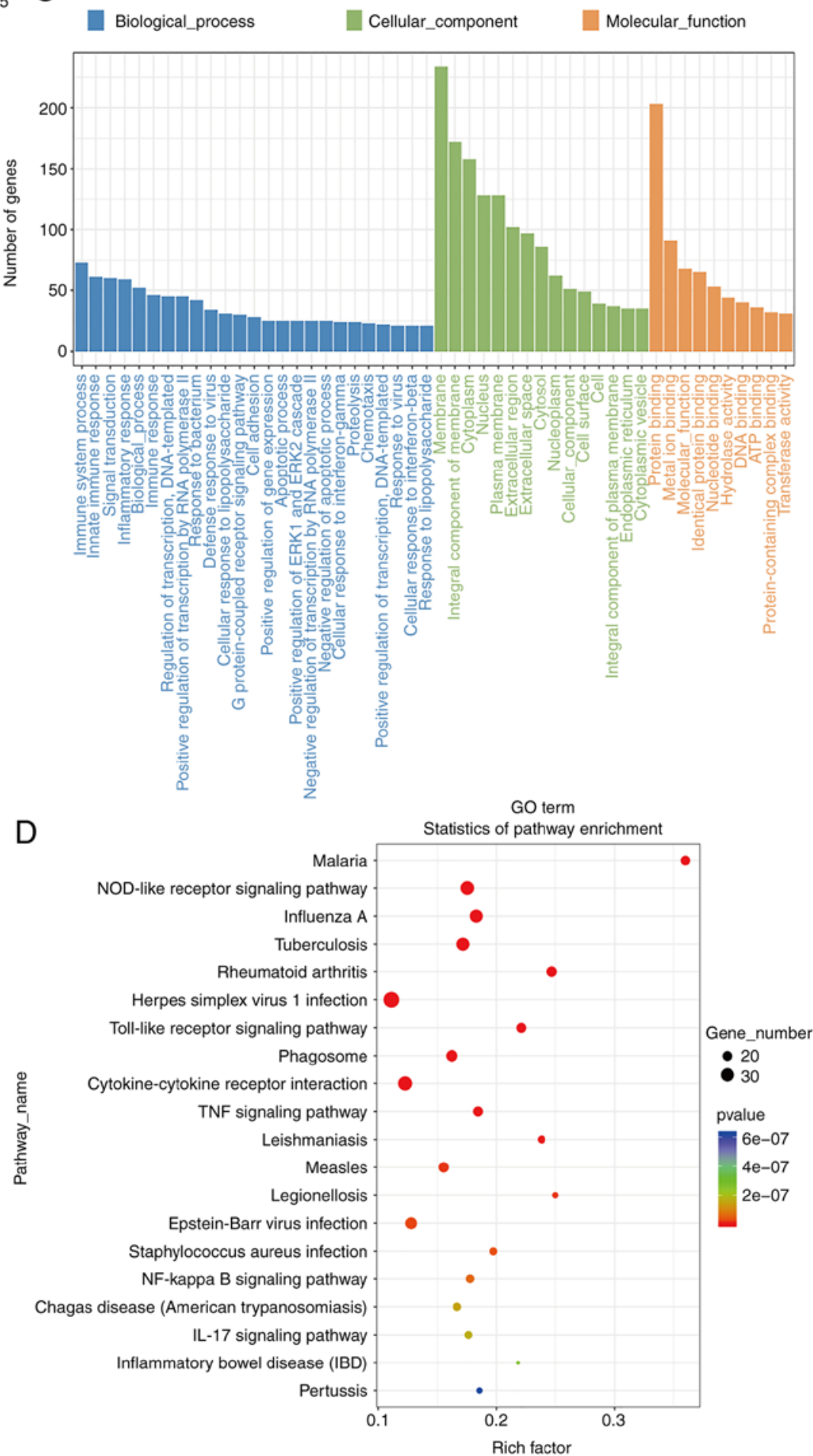

Figure 5. RNA sequencing transcriptional analysis of hippocampal mRNA from the sham and OVX groups. (A) Cluster analysis of DEGs. (B) Column chart analysis of DEGs. (C) Histogram of Gene Ontology functional term enrichment analysis results. (D) Lianchuan Biotechnology used ggplot2 to analyze the results of Kyoto Encyclopedia of Genes and Genomes signaling pathway enrichment analysis, and the results are presented as a scatter plot (n=3/group). OVX, ovariectomy; DEGs, differentially expressed genes. 

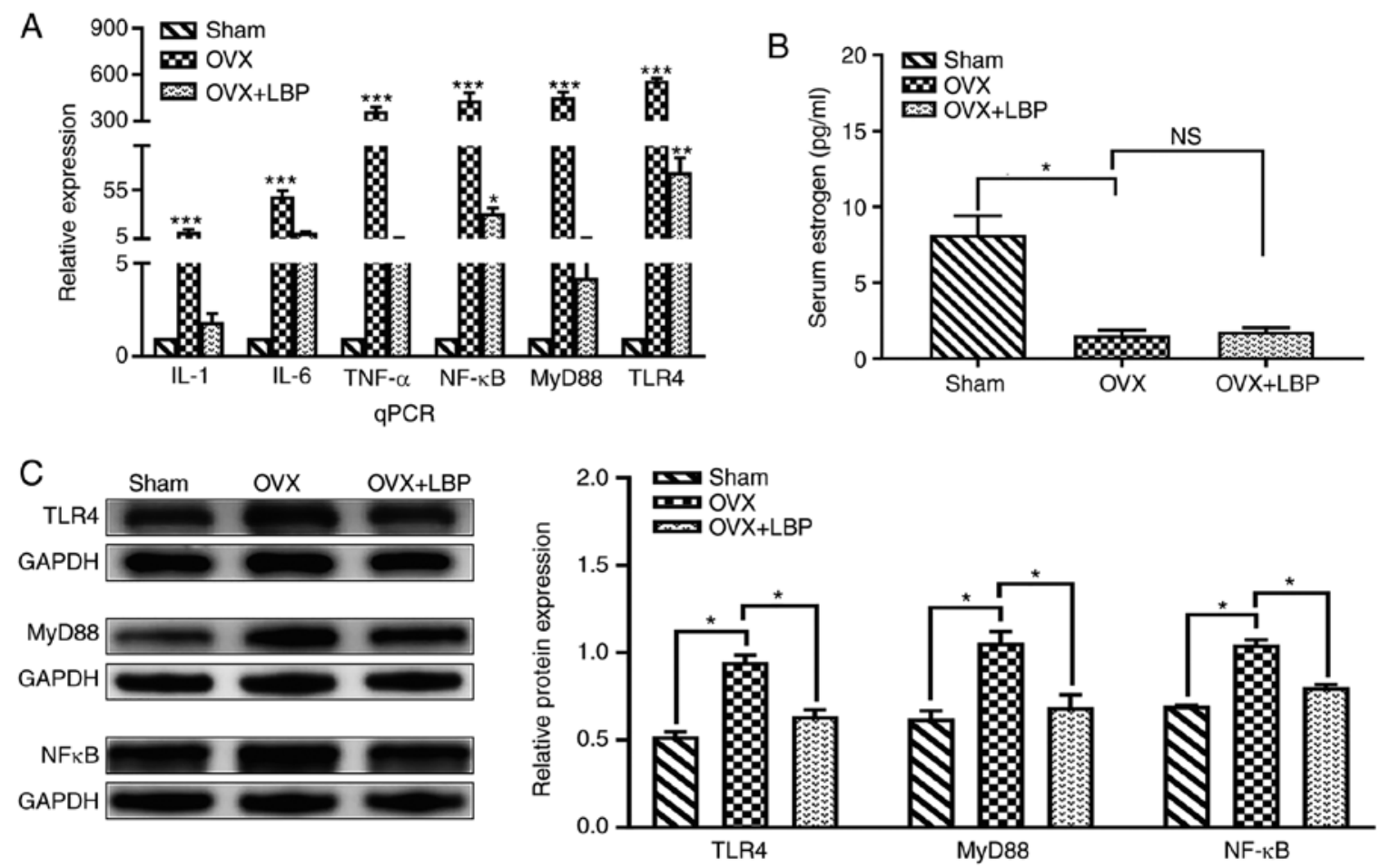

Figure 6. TLR4/NF-кB signaling pathway is closely associated with the progression of neuroinflammation in the brain. (A) Reverse transcription-quantitative PCR analysis of TLR4, MyD88, NFאB, TNF- $\alpha$, IL-1 $\beta$ and IL- 6 mRNA expression levels in hippocampal CA1 and CA3 regions. Data were analyzed using a one-way ANOVA and an LSD post hoc test. ${ }^{*} \mathrm{P}<0.05,{ }^{* *} \mathrm{P}<0.01,{ }^{* * * *} \mathrm{P}<0.001$ vs. the Sham group. (B) ELISA analysis of serum estrogen levels. Data were analyzed using a one-way ANOVA and an LSD post hoc test. "P $<0.05$ vs. the OVX group; ${ }^{\text {NS }} \mathrm{P}>0.05$ vs. the OVX group. (C) Western blot analysis of TLR4, MyD88 and NF- $\mathrm{KB}$ protein expression levels in the hippocampus. Data were analyzed using a one-way ANOVA and an LSD post hoc test. "P<0.05, vs. the OVX group. TLR, Toll-like receptor; MyD88, myeloid differentiation primary response 88; OVX, ovariectomy; LBP. Lycium barbarum polysaccharide; NS, not significant.

tissue was performed. Cluster analysis (Fig. 5A), column chart (Fig. 5B), GO functional term enrichment (Fig. 5C) and KEGG signaling pathway enrichment (Fig. 5D) analyses of DEGs were conducted, and the results revealed that the most significant changes were in inflammatory genes and inflammation-related pathways (Fig. 5). GO functional enrichment analysis revealed that the number of DEGs in the 'membrane', 'membrane components' and 'cytoplasm' was increased in OVX model mice compared with the sham mice (Fig. 5C). Among molecular functions, 'protein binding-related gene enrichment' was the most enriched. However, among biological processes, those associated with 'immune system processes', 'intracellular immune responses', 'signal transduction' and 'inflammatory response-related genes' exhibited more significant changes compared with other processes (Fig. 5C). KEGG signaling pathway enrichment analysis revealed that the most significant DEGs were those involved in 'nucleotide-binding', 'oligomerization domain (NOD)-like receptor signaling pathway', 'Toll-like receptor signaling pathway', 'cytokine-cytokine receptor interaction', 'TNF signaling pathway', 'NF-kB signaling pathway' and 'Epstein-Barr virus infection'(Fig. 5D).

Effect of LBP treatment on the expression levels of TLR4, $M y D 88$ and $N F-\kappa B$ in OVX model mice. To investigate the role of TLR4 signaling pathway in OVX-induced cognitive impairment, the mRNA expression levels of TLR4, MyD88 and $\mathrm{NF}-\mathrm{kB}$ in the hippocampus of sham, OVX and OVX + LBP mice were analyzed. The mRNA expression levels of TLR4,
MyD88 and NF- $\kappa B$ in the hippocampus were significantly upregulated in the OVX group compared with the sham group; however, the expression levels were significantly downregulated in the OVX + LBP group compared with the OVX group (Fig. 6A). The results of the ELISAs revealed that the serum estrogen levels were significantly reduced in mice following bilateral resection of the ovaries compared with the sham group. After oral LBP treatment, no significant difference was observed in the estrogen levels compared with the OVX group (P>0.05; Fig. 6B). These results indicated that the OVX was successful. In addition, the results of the western blotting revealed that the protein expression levels of TLR4, MyD88 and NF- $\kappa \mathrm{B}$ were significantly upregulated in the hippocampus of OVX model mice compared with the sham group; however, LBP treatment attenuated these increases (Fig. 6C).

Statistical analysis of the immunohistochemical findings (Fig. 7A and B) for TLR4, MyD88, and NF- $\kappa \mathrm{B}$ expression revealed that the number of positive cells in the hippocampal CA1 and CA 3 regions was significantly increased in the OVX group compared with the sham group. Notably, the number of positive cells in the OVX + LBP group was lower compared with the OVX group (Fig. 7C).

Effect of LBP treatment on the expression of inflammatory factors in OVX model mice. To further clarify the effect of LBP on brain inflammation in OVX model mice, the expression of key inflammatory proteins (IL6, IL-1 $\beta$ and TNF- $\alpha$ ) in the hippocampus were analyzed. As revealed in Fig. 7D, the expression levels of these three factors in the hippocampal 


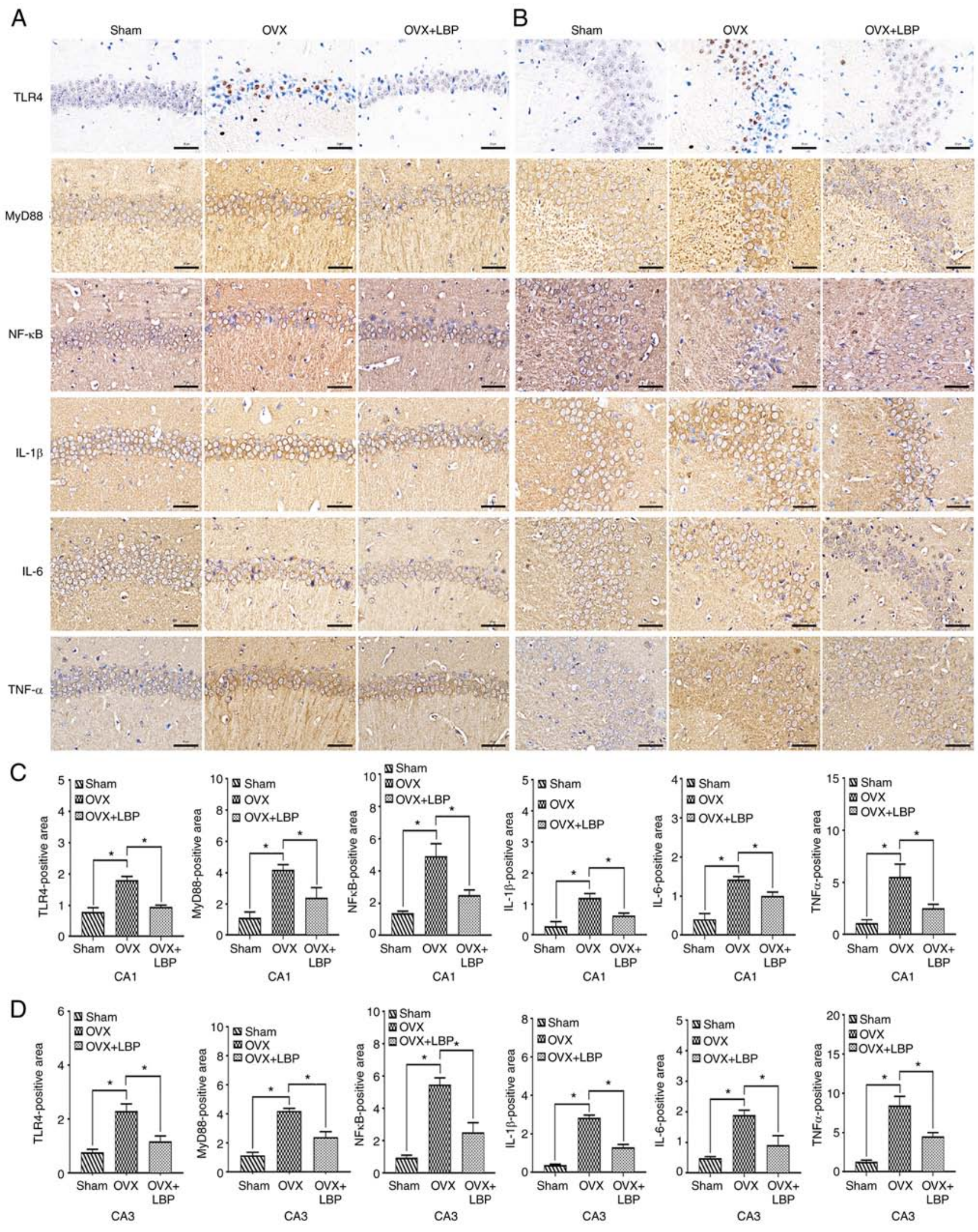

Figure 7. LBP inhibits the release of inflammatory factors and reduces neuroinflammation in OVX model mice. (A and B) Immunohistochemical staining was used to analyze the expression of TLR4, MyD88, NF- $\kappa$ B, IL-6, IL-1 $\beta$ and TNF- $\alpha$ in the hippocampal CA1 and CA3 regions. Scale bar, $20 \mu \mathrm{m}$.

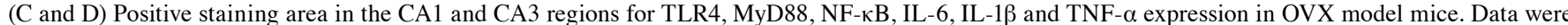
analyzed using a one-way ANOVA and an LSD post hoc test. " $\mathrm{P}<0.05$ vs. the OVX group ( $\mathrm{n}=5 /$ group). LBP, Lycium barbarum polysaccharide; OVX, ovariectomy; TLR, Toll-like receptor; MyD88, myeloid differentiation primary response 88.

CA1 and CA3 regions were significantly upregulated in the OVX group compared with the sham group. Compared with the OVX group mice, those treated with LPS exhibited downregulated expression levels of IL-6, IL-1 $\beta$ and TNF- $\alpha$. These 
findings were consistent with those obtained from RT-qPCR analysis.

\section{Discussion}

Aging has been associated with a wide range of effects in the CNS, including decreased cognitive ability, increased oxidative stress, and chronic inflammation (39). In the present study, an OVX-induced model was established to investigate the therapeutic effect of LBP on learning and memory impairments associated with estrogen deficiency, and the potential mechanisms underlying the observed effects were determined. The presents results suggested that LBP may attenuate learning and memory impairments and protect against hippocampal neuron injury. Furthermore, LBP could suppress the release of inflammatory factors and reduce neuroinflammation by downregulating the expression levels of mRNA and proteins associated with the TLR4/NF- $\kappa \mathrm{B}$ signaling pathway and decreasing the release of the proinflammatory cytokines IL-6, IL- $1 \beta$ and TNF- $\alpha$.

Impairments in cognition, learning and memory are the main clinical manifestations of neurodegenerative diseases (40). Cognitive decline caused by estrogen deficiency and other aging-related processes has become increasingly common given the rapid aging of the global population (41-44). Moreover, decreases in cognitive function were demonstrated to severely impact the quality of life of patients (45). Although effective interventions to address these impairments and their underlying mechanisms remain to be identified $(3,46)$, accumulating evidence has suggested that neuroinflammation in the brain may play a vital role in the pathophysiology of cognitive decline (47). To determine whether the anti-inflammatory properties of LBP exerted a protective effect against neurodegenerative disease, an OVX-induced cognitive impairment in vivo model was established, which is widely accepted as an experimental model for the study of neurodegenerative diseases $(47,48)$.

The results of the present study revealed that serum estrogen levels were significantly reduced following bilateral ovarian ablation and that this could not be attenuated by LBP treatment, which suggested that the OVX operation was successful and that oral LBP treatment did not affect serum estrogen levels. Subsequently, a novel target recognition testing method was used to evaluate spatial learning and memory in experimental animals. The present results indicated that the DI values were negative and the PI values were significantly decreased in OVX mice. These findings were consistent with previous data regarding the effects of OVX on spatial learning ability of mice (48). LBP treatment significantly increased PI and DI values and enhanced the spatial learning ability. In addition, the findings of the open-field test demonstrated that LBP treatment could improve OVX-induced impairments on motor ability. These results indicated that LBP could attenuate the effects of estrogen deficiency on spatial learning and memory in aging mice.

Damage to neurons in the hippocampal CA1 and CA3 regions was reported to induce impairments in learning and memory $(49,50)$. Notably, the hippocampus has been identified as a target for estrogen (51); however, to be the best of our knowledge, the reason for the estrogen-related neurochemical changes in the hippocampus remains unknown. The results of the present study indicated that the effect of LBP on learning and memory impairment may be associated with its protective effect on hippocampal neurons. To verify this hypothesis, $H \& E$ and Nissl staining were used to evaluate the effect of LBP on hippocampal neurons. The results indicated that oral LBP exerted a neuroprotective effect, significantly reducing the atrophy and loss of hippocampal neurons. In addition, the number of living neurons was negatively correlated with cognitive impairment. These findings suggest that LBP treatment may attenuate OVX-induced injury to hippocampal neurons and protect against impairments in learning and memory.

OVX modeling was previously revealed to induce neuroinflammation in the rodent brain, leading to impairments in learning, memory and cognition $(32,52,53)$. Further research indicated that OVX increased TLR2, TL4R and proinflammatory cytokine IL-6 levels, in addition to the activity of NF- $\kappa$ B in the hippocampus (54). Notably, several studies have reported that inhibiting the activation of TLR4 in the hippocampus could alleviate cognitive impairment (55-59). In addition, LBP could inhibit lipopolysaccharide-induced inflammation by blocking the TLR4/NF- $\kappa \mathrm{B}$ signaling pathway in RAW264.7 cells (60). LBP also reduced liver inflammation induced by $\mathrm{CCl}_{4}$ in Wistar rats by downregulating the expression levels of components of the TLR4/NF- $\kappa \mathrm{B}$ signaling pathway (61). To further investigate whether LBP plays a neuroprotective role in OVX model mice by regulating the TLR4/NF- $\mathrm{BB}$ signaling pathway, the present study analyzed the expression levels of TLR4, MyD88 and NF- $\mathrm{NB}$ in the CA1 and CA3 regions of the hippocampus using immunohistochemistry, RT-qPCR and western blotting. These analyses revealed that LBP downregulated the expression levels of all three of these factors, suggesting that LBP may reduce hippocampal inflammation by inhibiting the TLR4/NF- $\kappa \mathrm{B}$ signaling pathway.

Perivascular edema appeared in the images of certain figures, possibly caused by the immersion-fixation methodology that was used for immunohistochemistry. The use of immersion-fixation of the brain may not provide a thorough and suitable fixation which frequently leads to false results due to the loss and/or decrease and/or change of localization of the proteins and antigens in question. However, the mice brains are considerably small compared to the other experimental animals. In addition, the brain was fixed in $4 \%$ paraformaldehyde for $24 \mathrm{~h}$ at $4^{\circ} \mathrm{C}$ which may also facilitate the fixation to an extent. However, in the future study, perfusion-fixation will be considered to avoid artifacts.

In addition to inducing neuronal damage in the brain, OVX was revealed to activate astrocytes and microglia in the hippocampus and increased the release of inflammatory cytokines such as IL-6, IL-1 $\beta$ and TNF- $\alpha$, leading to decreases in cognitive function $(47,54,62)$. The present experimental results revealed that IL- 6 , IL- $1 \beta$ and TNF- $\alpha$ levels were significantly decreased following LBP treatment, further supporting the theory that LBP may reduce neuroinflammation. However, further studies are required to determine whether LBP exerts its effects via astrocytes or microglia.

In conclusion, the findings of the present study suggested that oral LBP may significantly attenuate OVX-induced learning 
and memory impairments in mice by blocking injury to hippocampal neurons via reducing neuroinflammation. Therefore, LBP may represent a potential therapeutic agent for the prevention and treatment of learning and memory impairments in patients with accelerated aging caused by ovarian dysfunction.

\section{Acknowledgements}

Not applicable.

\section{Funding}

The present study was supported by the National Natural Science Foundation of China (grant nos. 82060223 and 81460220), and the Ningxia Natural Science Foundation (grant nos. 2020AAC03143 and 2020AAC03150).

\section{Availability of data and materials}

The datasets used and/or analyzed during the present study are available from the corresponding author on reasonable request.

\section{Authors' contributions}

YW, ZM and YuL designed the study. XZ, JW, FB, JX and ZC performed the research. XZ and YiL analyzed the data. YW and $\mathrm{ZM}$ wrote and revised the manuscript. All authors read and approved the final manuscript.

\section{Ethics approval and consent to participate}

Guidelines for the Protection and Use of Experimental Animals (National Research Council of the National Academies), and approved by the Animal Ethics Committee of General Hospital of Ningxia Medical University, Ningxia, China (approval no. 2020-449).

\section{Patient consent for publication}

Not applicable.

\section{Competing interests}

The authors declare that they have no competing interests.

\section{References}

1. Kumar A: Editorial: Neuroinflammation and cognition. Front Aging Neurosci 10: 413, 2018.

2. Russell JK, Jones CK and Newhouse PA: The role of estrogen in brain and cognitive aging. Neurotherapeutics 16: 649-665, 2019.

3. Chen Z, Tang Y, Liu A, Jin X, Zhu J and Lu X: Oral administration of Grifola Frondosa polysaccharides improves memory impairment in aged rats via antioxidant action. Mol Nutr Food Res 61: 1700313, 2017.

4. Ryan J, Carriere I, Scali J, Ritchie K and Ancelin ML: Life-time estrogen exposure and cognitive functioning in later life. Psychoneuroendocrino 34: 287-298, 2009.

5. Lamar M, Resnick SM and Zonderman AB: Longitudinal changes in verbal memory in older adults: Distinguishing the effects of age from repeat testing. Neurology 60: 82-86, 2003.

6. Hohman TJ, Beason-Held LL, Lamar M and Resnick SM: Subjective cognitive complaints and longitudinal changes in memory and brain function. Neuropsychology 25: 125-130, 2011.
7. Campisi J, Kapahi P, Lithgow GJ, Melov S, Newman JC and Verdin E: From discoveries in ageing research to therapeutics for healthy ageing. Nature 571: 183-192, 2019.

8. Garbers C, Kuck F, Aparicio-Siegmund S, Konzak K, Kessenbrock M, Sommerfeld A, Häussinger D, Lang PA, Brenner D, Mak TW, et al: Cellular senescence Or EGFR signaling induces interleukin 6 (IL-6) receptor expression controlled by mammalian target of rapamycin (mTOR). Cell Cycle 12: 3421-3432, 2013.

9. Franceschi C, Garagnani P, Parini P, Giuliani C and Santoro A: Inflammaging: A new immune-metabolic viewpoint for age-related diseases. Nat Rev Endocrinol 14: 576-590, 2018.

10. Renz H, Holt PG, Inouye M, Logan AC, Prescott SL and Sly PD: An exposome perspective: Early-life events and immune development in a changing world. J Allergy Clin Immunol 140: 24-40, 2017.

11. Sly PD, Carpenter DO, Van den Berg M, Stein RT, Landrigan PJ, Brune-Drisse MN and Suk W: Health consequences of environmental exposures: Causal thinking in global environmental epidemiology. Ann Glob Health 82: 3-9, 2016.

12. Floreani A, Leung PS and Gershwin ME: Environmental basis of autoimmunity. Clin Rev Allergy Immunol 50: 287-300, 2016.

13. Ferrucci L and Fabbri E: Inflammageing: Chronic inflammation in ageing, cardiovascular disease, and frailty. Nat Rev Cardiol 15: 505-522, 2018.

14. Liberale L, Montecucco F, Tardif JC, Libby P and Camici GG: Inflamm-ageing: The role of inflammation in age-dependent cardiovascular disease. Eur Heart J 41: 2974-2982, 2020.

15. Franceschi $\mathrm{C}$ and Campisi J: Chronic inflammation (inflammaging) and its potential contribution to age-associated diseases. J Gerontol A Biol Sci Med Sci 69 (Suppl 1): S4-S9, 2014.

16. Wang RP, Ho YS, Leung WK, Goto T and Chang RC: Systemic inflammation linking chronic periodontitis to cognitive decline. Brain Behav Immun 81: 63-73, 2019.

17. Baulch JE, Acharya MM, Allen BD, Ru N, Chmielewski NN, Martirosian V, Giedzinski E, Syage A, Park AL, Benke SN, et al: Cranial grafting of stem cell-derived microvesicles improves cognition and reduces neuropathology in the irradiated brain. Proc Natl Acad Sci USA 113: 4836-4841, 2016.

18. Lee JY, Joo B, Nam JH, Nam HY, Lee W, Nam Y, Seo Y, Kang HJ, Cho HJ, Jang YP, et al: An aqueous extract of herbal medicine ALWPs enhances cognitive performance and inhibits LPS-induced neuroinflammation via FAK/NF-kappaB signaling pathways. Front Aging Neurosci 10: 269, 2018.

19. Jacobs AH and Tavitian B: Noninvasive molecular imaging of neuroinflammation. J Cereb Blood Flow Metab 32: 1393-1415, 2012.

20. Chandrasekaran A, Idelchik M and Melendez JA: Redox control of senescence and age-related disease. Redox Biol 11: 91-102, 2017.

21. Zeng P, Li J, Chen Y and Zhang L: The structures and biological functions of polysaccharides from traditional chinese herbs. Prog Mol Biol Transl Sci 163: 423-444, 2019.

22. Li S, Liu H, Wang W, Wang X, Zhang C, Zhang J, Jing H, Ren Z, Gao Z, Song X and Jia L: Antioxidant and anti-aging effects of acidic-extractable polysaccharides by agaricus bisporus. Int J Biol Macromol 106: 1297-1306, 2018.

23. Zhang R, Zhang X, Tang Y and Mao J: Composition, isolation, purification and biological activities of Sargassum Fusiforme Polysaccharides: A review. Carbohydr Polym 228: 115381, 2020.

24. Zhu Y, Yu X, Ge Q, Li J, Wang D, Wei Y and Ouyang Z: Antioxidant and anti-aging activities of polysaccharides from Cordyceps cicadae. Int J Biol Macromol 157: 394-400, 2020.

25. Li H, Li Z, Peng L, Jiang N, Liu Q, Zhang E, Liang B, Li R and Zhu H: Lycium barbarum polysaccharide protects human keratinocytes against UVB-induced photo-damage. Free Radic Res 51: 200-210, 2017.

26. Tian X, Liang T, Liu Y, Ding G, Zhang F and Ma Z: Extraction, structural characterization, and biological functions of Lycium Barbarum Polysaccharides: A Review. Biomolecules 9: 389, 2019.

27. Chang RC and So KF: Use of anti-aging herbal medicine, Lycium barbarum, against aging-associated diseases. What do we know so far? Cell Mol Neurobiol 28: 643-652, 2008.

28. Zhang Z, Zhou Y, Fan H, Billy KJ, Zhao Y, Zhan X, Yang L and Jia Y: Effects of Lycium barbarum polysaccharides on health and aging of C. Elegans depend On Daf-12/Daf-16. Oxid Med Cell Longev 2019: 6379493, 2019. 
29. Blair JA, Bhatta S and Casadesus G: CNS luteinizing hormone receptor activation rescues ovariectomy-related loss of spatial memory and neuronal plasticity. Neurobiol Aging 78: 111-120, 2019.

30. Bohm-Levine N, Goldberg AR, Mariani M, Frankfurt M and Thornton J: Reducing luteinizing hormone levels after ovariectomy improves spatial memory: Possible role of brain-derived neurotrophic factor. Horm Behav 118: 104590, 2020.

31. Monthakantirat O, Sukano W, Umehara K, Noguchi $H$, Chulikhit Y and Matsumoto K: Effect of miroestrol on ovariectomy-induced cognitive impairment and lipid peroxidation in mouse brain. Phytomedicine 21: 1249-1255, 2014.

32. Sbisa AM, Gogos A and van den Buuse M: Spatial working memory in the touchscreen operant platform is disrupted in female rats by ovariectomy but not estrous cycle. Neurobiol Learn Mem 144: 147-154, 2017.

33. Robinson MD, McCarthy DJ and Smyth GK: EdgeR: A bioconductor package for differential expression analysis of digital gene expression data. Bioinformatics 26: 139-140, 2010.

34. Pertea M, Pertea GM, Antonescu CM, Chang TC, Mendell JT and Salzberg SL: StringTie enables improved reconstruction of a transcriptome from RNA-seq reads. Nat Biotechnol 33: 290-295, 2015.

35. The Gene Ontology Resource: 20 years and still GOing strong. Nucleic Acids Res 47: D330-D338, 2019.

36. Kanehisa M, Furumichi M, Tanabe M, Sato Y and Morishima K KEGG: New perspectives on genomes, pathways, diseases and drugs. Nucleic Acids Res 45: D353-D361, 2017.

37. Livak KJ and Schmittgen TD: Analysis of relative gene expression data using real-time quantitative PCR and the 2(-Delta Delta C(T)) method. Methods 25: 402-408, 2001.

38. Shi Z, Zhu L, Li T, Tang X, Xiang Y, Han X, Xia L, Zeng L, Nie J, Huang Y, et al: Neuroprotective mechanisms of Lycium barbarum polysaccharides against ischemic insults by regulating NR2B and NR2A containing NMDA receptor signaling pathways. Front Cell Neurosci 11: 288, 2017.

39. Fjell AM, McEvoy L, Holland D, Dale AM and Walhovd KB What is normal in normal aging? Effects of aging, amyloid and Alzheimer's disease on the cerebral cortex and the hippocampus. Prog Neurobiol 117: 20-40, 2014.

40. Stanojlovic M,Pallais JP, Lee MK and Kotz CM: Pharmacological and chemogenetic orexin/hypocretin intervention ameliorates hipp-dependent memory impairment in the A53T mice model of Parkinson's disease. Mol Brain 12: 87, 2019.

41. No authors listed: 2020 Alzheimer's disease facts and figures. Alzheimers Dement: Mar 10, 2020 (Epub ahead of print).

42. Boyle PA, Yu L, Wilson RS, Leurgans SE, Schneider JA and Bennett DA: Person-specific contribution of neuropathologies to cognitive loss in old age. Ann Neurol 83: 74-83, 2018.

43. Nascimento C, Di Lorenzo Alho AT, Bazan Conceição Amaral C, Leite REP, Nitrini R, Jacob-Filho W, Pasqualucci CA, Hokkanen SRK, Hunter S, Keage H, et al: Prevalence of transactive response DNA-binding Protein 43 (TDP-43) proteinopathy in cognitively normal older adults: systematic review and meta-analysis. Neuropathol Appl Neurobiol 44: 286-297, 2018.

44. Morgan KN, Derby CA and Gleason CE: Cognitive changes with reproductive aging, perimenopause, and menopause. Obstet Gynecol Clin North Am 45: 751-763, 2018.

45. Bettio L, Rajendran L and Gil-Mohapel J: The effects of aging in the hippocampus and cognitive decline. Neurosci Biobehav Rev 79: 66-86, 2017.

46. Rehman SU, Shah SA, Ali T, Chung JI and Kim MO: Anthocyanins reversed D-galactose-induced oxidative stress and neuroinflammation mediated cognitive impairment in adult rats. Mol Neurobiol 54: 255-271, 2017.

47. Huang $\mathrm{C}$, Irwin MG, Wong $\mathrm{G}$ and Chang R: Evidence of the impact of systemic inflammation on neuroinflammation from a non-bacterial endotoxin animal model. J Neuroinflammation 15 : $147,2018$.
48. Liang J, Wu Y, Yuan H, Yang Y, Xiong Q, Liang C, Li Z, Li C, Zhang G, Lai X, et al: Dendrobium officinale polysaccharides attenuate learning and memory disabilities via anti-oxidant and anti-inflammatory actions. Int J Biol Macromol 126: 414-426, 2019.

49. Zhang X, Bai L, Zhang S, Zhou X, Li Y and Bai J: Trx-1 ameliorates learning and memory deficits in MPTP-induced Parkinson's disease model in mice. Free Radic Biol Med 124: 380-387, 2018

50. Arroyo-García LE, Tendilla-Beltrán H, Vázquez-Roque RA, Jurado-Tapia EE, Díaz A, Aguilar-Alonso P, Brambila E, Monjaraz E, De La Cruz F, Rodríguez-Moreno A and Flores G: Amphetamine sensitization alters hippocampal neuronal morphology and memory and learning behaviors. Mol Psychiatry: Jun 17, 2020 (Epub ahead of print)

51. Ramani M, Kumar R, Halloran B, Lal CV, Ambalavanan N and McMahon LL: Supraphysiological levels of oxygen exposure during the neonatal period impairs signaling pathways required for learning and memory. Sci Rep 8: 9914, 2018.

52. Lu J, Xu Y, Hu W, Gao Y, Ni X, Sheng H and Liu Y: Exercise ameliorates depression-like behavior and increases hippocampal BDNF level in ovariectomized rats. Neurosci Lett 573: 13-18, 2014.

53. Kouhestani S, Jafari A and Babaei P: Kaempferol attenuates cognitive deficit via regulating oxidative stress and neuroinflammation in an ovariectomized rat model of sporadic dementia. Neural Regen Res 13: 1827-1832, 2018.

54. Saied NM, Georgy GS, Hussien RM and Hassan WA: Neuromodulatory effect of curcumin on catecholamine systems and inflammatory cytokines in ovariectomized female rats. Clin Exp Pharmacol Physiol 48: 337-346, 2021.

55. Lu SM, Gui B, Dong HQ, Zhang X, Zhang SS, Hu LQ, Liu HL, Sun J and Qian YN: Prophylactic lithium alleviates splenectomy-induced cognitive dysfunction Possibly by inhibiting hippocampal TLR4 activation in aged rats. Brain Res Bull 114: 31-41, 2015.

56. Zhong Q, Zou Y, Liu H, Chen T, Zheng F, Huang Y, Chen C and Zhang Z: Toll-like receptor 4 deficiency ameliorates $\beta 2$-microglobulin induced age-related cognition decline due to neuroinflammation in mice. Mol Brain 13: 20, 2020.

57. Wang S,Zhang X,Zhai L, Sheng X, Zheng W, Chu H and Zhang G: Atorvastatin attenuates cognitive deficits and neuroinflammation induced by $\mathrm{A} \beta_{1-42}$ involving modulation of TLR4/TRAF6/NF- $\kappa \mathrm{B}$ pathway. J Mol Neurosci 64: 363-373, 2018.

58. Zhang Q, Wu HH, Wang Y, Gu GJ, Zhang W and Xia R: Neural stem cell transplantation decreases neuroinflammation in a transgenic mouse model of Alzheimer's disease. J Neurochem 136: 815-825, 2016.

59. He P, Yan S, Zheng J, Gao Y, Zhang S, Liu Z, Liu X and Xiao C: Eriodictyol attenuates LPS-induced neuroinflammation, amyloidogenesis, and cognitive impairments via the inhibition of $\mathrm{NF}-\kappa \mathrm{B}$ in male C57BL/6J mice and BV2 microglial cells. J Agric Food Chem 66: 10205-10214, 2018.

60. Xu Y, Sheng H, Bao Q, Wang Y, Lu J and Ni X: NLRP3 inflammasome activation mediates estrogen deficiency-induced depression- and anxiety-like behavior and hippocampal inflammation in mice. Brain Behav Immun 56: 175-186, 2016.

61. Peng Q, Liu H, Shi S and Li M: Lycium ruthenicum polysaccharide attenuates inflammation through inhibiting TLR4/NF- $\mathrm{BB}$ signaling pathway. Int J Biol Macromol 67: 330-335, 2014.

62. Gan F, Liu Q, Liu Y, Huang D, Pan C, Song S and Huang K: Lycium barbarum polysaccharides improve CCl4-induced liver fibrosis, inflammatory response and TLRs/NF-kB signaling pathway expression in wistar rats. Life Sci 192: 205-212, 2018.

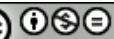

This work is licensed under a Creative Commons Attribution-NonCommercial-NoDerivatives 4.0 International (CC BY-NC-ND 4.0) License. 